\title{
Simple Method of the Formation of the Hamiltonian Matrix for Some Schrödinger Equations Describing the Molecules with Large Amplitude Motions
}

\author{
George A. Pitsevich, Alex E. Malevich \\ Belarusian State University, Minsk, Belarus \\ Email: pitsevich@bsu.by
}

Received September 8, 2012; revised October 7, 2012; accepted October 18, 2012

\begin{abstract}
A simple approach to the formation of a Hamiltonian matrix for some Schrödinger equations describing the molecules with large amplitude motions has been proposed. The algorithm involving one or several variables has been concretely defined for the basis functions represented by Fourier series and orthogonal polynomials, taking Hermitian polynomials as an example.
\end{abstract}

Keywords: Schrödinger Equation; Large Amplitude Motions; Hamiltonian Matrix

\section{Introduction}

Algebraic approaches to solving of Schrödinger equations have several advantages compared to other methods. Provided the basis functions used for expansion of the wave functions and the potential energy of a system are adequately selected, the Schrödinger equation takes the matrix form, in the end its solution being reduced to the derivation of eigenfunctions and eigenvalues for the Hamiltonian matrix. When studying the molecules whose variables are changing with a large amplitude, the Hamiltonian matrix derivation is a nontrivial problem. This work presents an algorithm to form the Hamiltonian matrix for some Schrödinger equations describing molecules and molecular systems with several variables of this type. Such equations may be illustrated by the following:

$$
\begin{aligned}
& -F \frac{\mathrm{d}^{2} \Psi}{\mathrm{d} \varphi^{2}}+U(\varphi)=E \Psi \\
& -A \frac{\partial^{2} \Psi(s, t)}{\partial s^{2}}-B \frac{\partial^{2} \Psi(s, t)}{\partial t^{2}}+U(s, t) \Psi(s, t) \\
& =E \Psi(s, t) \\
& -F\left(\frac{\partial^{2} \Psi(x, y, z)}{\partial x^{2}}+\frac{\partial^{2} \Psi(x, y, z)}{\partial y^{2}}+\frac{\partial^{2} \Psi(x, y, z)}{\partial z^{2}}\right) \\
& +U(x, y, z) \Psi(x, y, z)=E \Psi(x, y, z)
\end{aligned}
$$

In all cases it is assumed that with a change in the vari- ables the kinematic parameters remain invariable. This may be attained by an adequate selection of a coordinate system strongly related to the molecule, both in the case of symmetric [1-4] and low-symmetry [5-9] molecules. Equation (1), in particular, describes internal vibrations in a molecule of methanol, taking the effective vibrational constant as $F$ [10-12]. In some cases an invariable character of the molecular kinematic parameters for large amplitude motions may be considered as a physically valid approximation. Specifically, Equation (3) may be used for the description of motion of a hydrogen atom in the process of hydrogen bonding if we neglect motion of the oxygen atoms, the amplitude of which is in fact considerably smaller than that of $H$ motion as a mass ratio of these atoms is 1:16. This paper presents "quick" approaches to construct the Hamiltonian matrix for some basis functions.

\section{Using of Fourier Series}

Since the use of Fourier series for solving of equations of the form given in (1) is frequently described in the literature and in some works the formation algorithm for the Hamiltonian matrix is given in detail, we begin our analysis from Equation (2). Let the potential energy be given in the form:

$$
U(s, t)=\sum_{k, l=-a,-b}^{a, b} u_{k l} \mathrm{e}^{\mathrm{i}(k s+l t)} ; \quad a, b \in \mathbb{N}
$$

Then a wave function is derived as: 


$$
\Psi(s, t)=\sum_{n, m=-\infty}^{\infty} b_{n m} \mathrm{e}^{\mathrm{i}(n s+m t)}
$$

Substituting (4) and (5) into (2), we obtain:

$$
\begin{aligned}
& \sum_{n, m=-\infty}^{\infty}\left(n^{2} A+m^{2} B-E\right) b_{n m} \mathrm{e}^{\mathrm{i}(n s+m t)} \\
& +\sum_{n, m=-\infty}^{\infty} \sum_{k, l=-a,-b}^{a, b} u_{k l} b_{n m} \mathrm{e}^{\mathrm{i}((n+k) s+(m+l) t)}=0
\end{aligned}
$$

Next we define coefficients for the exponential $\mathrm{e}^{\mathrm{i}\left(n^{\prime} s+m^{\prime} t\right)}$. In the second term the following condition must be fulfilled:

$$
\begin{aligned}
& n+k=n^{\prime} \Rightarrow k=n^{\prime}-n ; \\
& m+l=m^{\prime} \Rightarrow l=m^{\prime}-m
\end{aligned}
$$

Instead of (6), we have:

$$
\begin{aligned}
& \left(n^{\prime 2} A+m^{\prime 2} B-E\right) b_{n^{\prime} m^{\prime}} \mathrm{e}^{\mathrm{i}\left(n^{\prime} s+m^{\prime} t\right)} \\
& +\sum_{n, m=-\infty}^{\infty} \sum_{n^{\prime}-n, m^{\prime}-m=-a,-b}^{a, b} u_{n^{\prime}-n, m^{\prime}-m} b_{n m} \mathrm{e}^{\mathrm{i}\left(n^{\prime} s+m^{\prime} t\right)}=0
\end{aligned}
$$

Then we construct the finite matrix with the dimensions $(2 c+1)^{2} \times(2 c+1)^{2} ; c \in N$. This means that $n$ and $m$ are varying within the limits from $-c$ to $c$ per unity. From (8) we derive:

$$
\begin{aligned}
& \left(n^{\prime 2} A+m^{\prime 2} B-E\right) b_{n^{\prime} m^{\prime}} \mathrm{e}^{\mathrm{i}\left(n^{\prime} s+m^{\prime} t\right)} \\
& +\sum_{n, m=-c}^{c} \sum_{n^{\prime}-n, m^{\prime}-m=-a,-b}^{a, b} u_{n^{\prime}-n, m^{\prime}-m} b_{n m} \mathrm{e}^{\mathrm{i}\left(n^{\prime} s+m^{\prime} t\right)}=0
\end{aligned}
$$

Now we take (9) as a matrix equation of the form $H_{i j}\left\|b_{j}\right\|=E_{j}\left\|b_{j}\right\|$, where $\left\|b_{j}\right\|-$ column vector that, according to (5), gives the wave function corresponding to the energy $E_{j}$. It is clear that a pair of the indices $\left(n^{\prime}, m^{\prime}\right)$ numbers rows of the Hamiltonian matrix and a pair of the indices $(n, m)$-its columns. Next, to derive the Hamiltonian matrix from (9), first we have to fix an order of the coefficients $b_{n m}$ in the column vector of the wave function defined by Equation (5). For example, if $c=1$, the transposed column vector may be of the form:

$$
\|\tilde{b}\|=\left\langle b_{-1,-1} ; b_{-1,0} ; b_{-1,1} ; b_{0,-1} ; b_{0,0} ; b_{0,1} ; b_{1,-1} ; b_{1,0} ; b_{1,1}\right\rangle
$$

Let us assume that in the same order from top to bottom there is a change in the index pair $\left(n^{\prime}, m^{\prime}\right)$ numbering rows of the Hamiltonian matrix. Then a matrix element of $H$ is numbered by two index pairs, $H_{\left(n^{\prime}, m^{\prime}\right),(n, m)}$. Considering that usually $c \gg a, b$, for the diagonal element $\left(n=n^{\prime} ; m=m^{\prime}\right)$ we can write:

$$
H_{\left(n^{\prime}, m^{\prime}\right),\left(n^{\prime}, m^{\prime}\right)}=n^{\prime 2} A+m^{\prime 2} B+u_{00}-E
$$

and for nondiagional elements we can write:

$$
\begin{aligned}
& H_{\left(n^{\prime}, m^{\prime}\right),(n, m)}=u_{n^{\prime}-n, m^{\prime}-m} \\
& \text { if }\left|n^{\prime}-n\right| \leq a \text { and }\left|m^{\prime}-m\right| \leq b \\
& H_{\left(n^{\prime}, m^{\prime}\right),(n, m)}=0 \\
& \text { if }\left|n^{\prime}-n\right| \succ a \text { or }\left|m^{\prime}-m\right| \succ b
\end{aligned}
$$

Numbering matrix elements of $H$ by the ordinary indices $(i, j)$ each of which is varying from 1 to $(2 c+1)^{2}$, we should establish for each of them a oneto-one correspondence to a pair of numbers by the principle: $i \Leftrightarrow\left(n_{i}^{\prime}, m_{i}^{\prime}\right) ; j \Leftrightarrow\left(n_{j}, m_{j}\right)$. Specifically, in the case given by (10) for $i=3$ we have $n_{3}^{\prime}=-1 ; m_{3}^{\prime}=1$; and for $j=6$ we have $n_{6}=0 ; m_{6}=1$. Now an algorithm for the formation of the matrix $H$ takes the following form:

$$
\begin{aligned}
& H_{i i}=n_{i}^{\prime 2} A+m_{i}^{\prime 2} B+u_{00}-E \\
& H_{i j}=u_{n_{i}^{\prime}-n_{j}, m_{i}^{\prime}-m_{j}} \\
& \text { if }\left|n_{i}^{\prime}-n_{j}\right| \leq a \text { and }\left|m_{i}^{\prime}-m_{j}\right| \leq b \\
& H_{i j}=0 \text { if }\left|n_{i}^{\prime}-n_{j}\right| \succ a \text { or }\left|m_{i}^{\prime}-m_{j}\right| \succ b
\end{aligned}
$$

Let us write the Hamiltonian matrix in the explicit form with the use of $(14-16)$ for $c=1$. Besides, we assume that the index order is determined by the relation of (10), and $a=b=1$. Then we have:

$$
\begin{array}{ccccccccc}
A+B+u_{00} & u_{0,-1} & 0 & u_{-1,0} & u_{-1,-1} & 0 & 0 & 0 & 0 \\
u_{0,1} & A+u_{00} & u_{0,-1} & u_{-1,1} & u_{-1,0} & u_{-1,-1} & 0 & 0 & 0 \\
0 & u_{0,1} & A+B+u_{00} & 0 & u_{-1,1} & u_{-1,0} & 0 & 0 & 0 \\
u_{1,0} & u_{1,-1} & 0 & B+u_{00} & u_{0,-1} & 0 & u_{-1,0} & u_{-1,-1} & 0 \\
u_{1,1} & u_{1,0} & u_{1,-1} & u_{0,1} & u_{00} & u_{0,-1} & u_{-1,1} & u_{-1,0} & u_{-1,-1} \\
0 & u_{1,1} & u_{1,0} & 0 & u_{0,1} & B+u_{00} & 0 & u_{-1,1} & u_{-1,0} \\
0 & 0 & 0 & u_{1,0} & u_{1,-1} & 0 & A+B+u_{00} & u_{0,-1} & 0 \\
0 & 0 & 0 & u_{1,1} & u_{1,0} & u_{1,-1} & u_{0,1} & A+u_{00} & u_{0,-1} \\
0 & 0 & 0 & 0 & u_{1,1} & u_{1,0} & 0 & u_{0,1} & A+B+u_{00}
\end{array}
$$

Next we consider the case of three variables. Let the Schrödinger equation be of the form: 


$$
\begin{aligned}
& -A \frac{\partial^{2} \Psi(s, t, r)}{\partial s^{2}}-B \frac{\partial^{2} \Psi(s, t, r)}{\partial t^{2}}-C \frac{\partial^{2} \Psi(s, t, r)}{\partial r^{2}} \\
& +U(s, t, r) \Psi(s, t, r)=E \Psi(s, t, r)
\end{aligned}
$$

Then we define an algorithm to form the Hamiltonian matrix when using three-dimensional Fourier series. Let the potential energy be given as:

$$
U(s, t, r)=\sum_{h, k, l=-a,-b,-c}^{a, b, c} u_{h k l} \mathrm{e}^{\mathrm{i}(h s+k t+l r)} ;
$$$$
a, b, c \in N
$$

A wave function takes the form:

$$
\Psi(s, t, r)=\sum_{n, m, q=-\infty}^{\infty} b_{n m q} \mathrm{e}^{\mathrm{i}(n s+m t+q r)}
$$

Substituting (18) and (19) into (17), we obtain:

$$
\begin{aligned}
& \sum_{n, m, q=-\infty}^{\infty}\left(n^{2} A+m^{2} B+q^{2} C-E\right) b_{n m q} \mathrm{e}^{\mathrm{i}(n s+m t+q r)} \\
& +\sum_{n, m, q=-\infty}^{\infty} \sum_{h, k, l=-a,-b,-c}^{a, b, c} u_{h k l} b_{n m q} \mathrm{e}^{\mathrm{i}((n+h) s+(m+k) t+(l+q) r)}=0
\end{aligned}
$$

Let us find coefficients for the exponential $\mathrm{e}^{\mathrm{i}\left(n^{\prime} s+m^{\prime} t+q^{\prime} r\right)}$. The following condition must be fulfilled:

$$
\begin{aligned}
& n+h=n^{\prime} \Rightarrow h=n^{\prime}-n ; \\
& m+k=m^{\prime} \Rightarrow k=m^{\prime}-m ; \\
& l+q=l^{\prime} \Rightarrow q=l^{\prime}-l ;
\end{aligned}
$$

Instead of (20), we have:

$$
\begin{aligned}
& \left(n^{\prime 2} A+m^{\prime 2} B+q^{\prime 2} C-E\right) b_{n^{\prime} m^{\prime} q} \mathrm{e}^{\mathrm{i}\left(n^{\prime} s+m^{\prime} t+q^{\prime} r\right)} \\
& +\sum_{n, m, q=-\infty}^{\infty} \sum_{n^{\prime}-n, m^{\prime}-m, l^{\prime}-l=-a,-b,-c}^{a, b, c} u_{n^{\prime}-n, m^{\prime}-m, l^{\prime}-l} b_{n m q} \mathrm{e}^{\mathrm{i}\left(n^{\prime} s+m^{\prime} t+q^{\prime} r\right)} \\
& =0
\end{aligned}
$$

We construct the finite matrix with the dimensions $(2 d+1)^{3} \times(2 d+1)^{3} ; d \in N$, i.e. $n, m$, and $q$ are varying within the limits from $-d$ to $d$ per unity. From Equation (22) we get:

$$
\begin{aligned}
& \left(n^{\prime 2} A+m^{\prime 2} B+q^{\prime 2} C-E\right) b_{n^{\prime} m^{\prime} q^{\prime}} \mathrm{e}^{\mathrm{i}\left(n^{\prime} s+m^{\prime} t+q^{\prime} r\right)} \\
& +\sum_{n, m, q=-d}^{d} \sum_{n^{\prime}-n, m^{\prime}-m, l^{\prime}-l=-a,-b,-c}^{a, b, c} u_{n^{\prime}-n, m^{\prime}-m, l^{\prime}-l} b_{n m q} \mathrm{e}^{\mathrm{i}\left(n^{\prime} s+m^{\prime} t+l^{\prime} r\right)} \\
& =0
\end{aligned}
$$

Now three indices $\left(n^{\prime}, m^{\prime}, l^{\prime}\right)$ number rows and three indices $(n, m, l)$ number columns of the Hamiltonian matrix. To derive a Hamiltonian matrix from (23), we again fix an order of the coefficients $b_{\text {nmq }}$ in the column vector for the wave function defined by (19). For example, if $c=1$, the transposed column vector may be of the form:

$$
\begin{aligned}
\|\tilde{b}\|= & \left\langle b_{-1,-1,-1} ; b_{-1,-1,0} ; b_{-1,-1,1} ; b_{-1,0,-1} ; b_{-1,0,0} ;\right. \\
& b_{-1,0,1} ; b_{-1,1,-1} ; b_{-1,1,0} ; b_{-1,1,1} ; b_{0,-1,-1} ; b_{0,-1,0} ; \\
& b_{0,-1,1} ; b_{0,0,-1} ; b_{0,0,0} ; b_{0,0,1} ; b_{0,1,-1} ; b_{0,1,0} ; b_{0,1,1} ; \\
& b_{1,-1,-1} ; b_{1,-1,0} ; b_{1,-1,1} ; b_{1,0,-1} ; b_{1,0,0} ; b_{1,0,1} ; \\
& \left.b_{1,1,-1} ; b_{1,1,0} ; b_{1,1,1}\right\rangle
\end{aligned}
$$

We assume that a change of three indices $n^{\prime}, m^{\prime}, q^{\prime}$, numbering rows for the Hamiltonian matrix is in the same order from top to bottom. Then a matrix element of $H$ is numbered by two pairs of three indices, $H_{\left(n^{\prime}, m^{\prime}, q^{\prime}\right),(n, m, q)}$. Considering that, as previously, we have $d \gg a, b, c$, then for the diagonal elements $\left(n=n^{\prime} ; m=m^{\prime} ; q^{\prime}=q\right)$ we can write:

$$
H_{\left(n^{\prime}, m^{\prime}, q^{\prime}\right),\left(n^{\prime}, m^{\prime}, q^{\prime}\right)}=n^{\prime 2} A+m^{\prime 2} B+q^{\prime 2} C+u_{000}-E
$$

And for nondiagonal elements we can write:

$$
\begin{aligned}
& H_{\left(n^{\prime}, m^{\prime}, q^{\prime}\right),(n, m, q)}=u_{n^{\prime}-n, m^{\prime}-m, q^{\prime}-q} \\
& \text { if }\left|n^{\prime}-n\right| \leq a ;\left|m^{\prime}-m\right| \leq b \text { and }\left|q^{\prime}-q\right| \leq c \\
& H_{\left(n^{\prime}, m^{\prime}, q^{\prime}\right),(n, m, q)}=0 \text { if }\left|n^{\prime}-n\right| \succ a \\
& \text { or }\left|m^{\prime}-m\right| \succ b \text { or }\left|q^{\prime}-q\right| \succ c
\end{aligned}
$$

when numbering the matrix elements of $H$ by the ordinary indices $(i, j)$ each of which is varying from 1 to $(2 c+1)^{3}$, we should establish for each of them a one-to-one correspondence to three numbers by the principle: $i \Leftrightarrow\left(n_{i}^{\prime}, m_{i}^{\prime}, q_{i}^{\prime}\right) ; j \Leftrightarrow\left(n_{j}, m_{j}, q_{j}\right)$. Specifically, in the case given by (24) for $i=3$ we have $n_{3}^{\prime}=-1$; $m_{3}^{\prime}=-1 ; \quad q_{3}^{\prime}=1$, and for $j=19$ we have $n_{19}=1$; $m_{19}=-1 ; \quad q_{19}=-1$. Now an algorithm to form the matrix $H$ takes the form:

$$
\begin{aligned}
& H_{i i}=n_{i}^{\prime 2} A+m_{i}^{\prime 2} B+q_{i}^{\prime 2} C+u_{000}-E \\
& H_{i j}=u_{n_{i}^{\prime}-n_{j}, m_{i}^{\prime}-m_{j}, q_{i}^{\prime}-q_{j}} \text { if }\left|n_{i}^{\prime}-n_{j}\right| \leq a ; \\
& \left|m_{i}^{\prime}-m_{j}\right| \leq b \text { and }\left|q_{i}^{\prime}-q_{j}\right| \leq c \\
& H_{i j}=0 \text { if }\left|n_{i}^{\prime}-n_{j}\right| \succ a \\
& \text { or }\left|m_{i}^{\prime}-m_{j}\right| \succ b \text { or }\left|q_{i}^{\prime}-q_{j}\right| \succ c
\end{aligned}
$$

\section{Using of Orthogonal Polynomials}

Let us consider the Schrödinger equation with one variable (31), taking orthogonal Hermitian polynomials $H_{n}$ as an example.

$$
-R \frac{\mathrm{d}^{2} \Psi(x)}{\mathrm{d} x^{2}}+U(x) \Psi(x)=E \Psi(x)
$$

Let the potential energy be given as:

$$
U(x)=\sum_{k=0}^{m} u_{k} H_{k}(x)
$$


And we are looking for a wave function of the form:

$$
\Psi(x)=\sum_{n=0}^{\infty} b_{n} H_{n}(x) \mathrm{e}^{-\frac{1}{2} x^{2}}
$$

We substitute (32) and (33) into (31):

$$
\begin{aligned}
& \sum_{n=0}^{\infty}\left(R\left(n+\frac{1}{2}\right)-E\right) b_{n} H_{n}(x) \\
& -\sum_{n=0}^{\infty} R n(n-1) b_{n} H_{n-2}(x) \\
& -\sum_{n=0}^{\infty} \frac{R}{4} b_{n} H_{n+2}(x) \\
& +\sum_{n=0}^{\infty} \sum_{k=0}^{m} u_{k} b_{n} H_{n}(x) H_{k}(x)=0
\end{aligned}
$$

Using the orthogonality of Hermitian polynomials, we can write:

$$
\begin{aligned}
& H_{n}(x) H_{k}(x)=\sum_{l=|n-k|, 2}^{n+k} c_{l, n, k} H_{l}(x) \\
& C_{l, n, k}=\int_{-\infty}^{\infty} H_{n}(x) H_{k}(x) H_{l}(x) \mathrm{e}^{-\frac{1}{2} x^{2}} \mathrm{~d} x
\end{aligned}
$$

As a result, Equation (34) takes the form:

$$
\begin{aligned}
& \sum_{n=0}^{\infty}\left(R\left(n+\frac{1}{2}\right)-E\right) b_{n} H_{n}(x)-\sum_{n=0}^{\infty} R n(n-1) b_{n} H_{n-2}(x) \\
& -\sum_{n=0}^{\infty} \frac{R}{4} b_{n} H_{n+2}(x)+\sum_{n=0}^{\infty} \sum_{k=0}^{m} \sum_{l=|n-k|, 2}^{n+k} c_{l, n, k} u_{k} b_{n} H_{l}(x)=0
\end{aligned}
$$

Taking the coefficients for $H_{n^{\prime}}$, we construct a matrix with the dimensions $(h+1) \times(h+1)$. In the second term of Equation (36) we must assume

$$
n-2=n^{\prime} \Rightarrow n=n^{\prime}+2 \text {, }
$$

in the third term we assume $n+2=n^{\prime} \Rightarrow n=n^{\prime}-2$, and in the fourth $-l=n^{\prime}$. Instead of (36), we get:

$$
\begin{aligned}
& \left(R\left(n^{\prime}+\frac{1}{2}\right)-E\right) b_{n^{\prime}} H_{n^{\prime}}(x) \\
& -R\left(n^{\prime}+2\right)\left(n^{\prime}+1\right) b_{n^{\prime}+2} H_{n^{\prime}}(x) \\
& -\frac{R}{4} b_{n^{\prime}-2} H_{n^{\prime}}(x)+\sum_{n=0}^{h} \sum_{k} c_{n^{\prime} n k} H_{n^{\prime}}(x) b_{n} u_{k}=0
\end{aligned}
$$

As previously, the index $n^{\prime}$ numbers rows of the Hamiltonian matrix, whereas the index $n$ numbers its columns. Let an order of indices in the column vector of the wave function be so that a form of the transposed vector is given by:

$$
\|\tilde{b}\|=\left\langle b_{0} ; b_{1} ; \cdots b_{h}\right\rangle
$$

In a similar way we will number rows of the Hamiltonian matrix from top to bottom from 0 to $h$ per unity. According to Equation (37), at the first stage we can fill the Hamiltonian matrix with the elements existing for representation of the potential energy in the form $c_{n^{\prime} n k} u_{k}$ by the following principle:

$$
H_{n^{\prime} n}=\sum_{k} c_{n^{\prime} n k} u_{k}
$$

Summation in (39) is over all the existing indices $k$ for the specified index pair $\left(n^{\prime}, n\right)$. Next, to every diagonal element $H_{n^{\prime} n^{\prime}}$ we add $\left(n^{\prime}+\frac{1}{2}\right) R$ and to every element of the diagonal, parallel to the main diagonal and positioned above it as a next nearest $\left(H_{n^{\prime}, n^{\prime}+2}\right)$, we add $-\left(n^{\prime}+2\right)\left(n^{\prime}+1\right) R$. And in the case of a similar diagonal positioned as a next nearest below $\left(H_{n^{\prime}, n^{\prime}-2}\right)$ we add $-\frac{1}{4} R$. So, diagonal elements take the form:

$$
H_{n^{\prime} n^{\prime}}=\left(n^{\prime}+\frac{1}{2}\right) R+\sum_{k} c_{n^{\prime} n^{\prime} k} u_{k}
$$

Nondiagonal elements are of the form:

$$
\begin{aligned}
& H_{n^{\prime} n^{\prime}+2}=-\left(n^{\prime}+2\right)\left(n^{\prime}+1\right) R+\sum_{k} c_{n^{\prime}, n^{\prime}+2, k} u_{k} \\
& H_{n^{\prime} n^{\prime}-2}=-\frac{1}{4} R+\sum_{k} c_{n^{\prime}, n^{\prime}-2, k} u_{k}
\end{aligned}
$$

The remaining nondiagonal elements are as (39). Using the ordinary indices $i, j$ varying from 1 to $h+1$, we can rewrite this algorithm as:

$$
\begin{aligned}
& H_{i i}=\left(i-\frac{1}{2}\right) R+\sum_{k} c_{i-1, i-1, k} u_{k} \\
& H_{i, i+2}=-(i+1) i R+\sum_{k} c_{i-1, i+1, k} u_{k} \\
& H_{i, i-2}=-\frac{1}{4} R+\sum_{k} c_{i-1, i-3, k} u_{k} \\
& H_{i j}=\sum_{k} c_{i-1, j-1, k} u_{k}
\end{aligned}
$$

Finally, we consider Equation (3), trying to construct the Hamiltonian matrix with the use of Hermitian polynomials as basis functions. Let the potential energy be represented as:

$$
\begin{aligned}
& U(x, y, z)=\sum_{k, l, m=0}^{a, b, c} u_{k l m} H_{k}(x) H_{l}(y) H_{m}(z) ; \\
& a, b, c \in N
\end{aligned}
$$

A wave function is derived as follows:

$$
\begin{aligned}
& \Psi(x, y, z)=\sum_{n t s=0}^{\infty} b_{n t s} H_{n}(x) H_{t}(y) H_{s}(z) \mathrm{e}^{-\frac{r^{2}}{2}} ; \\
& r^{2}=x^{2}+y^{2}+z^{2}
\end{aligned}
$$

Substituting (47) and (48) into (3), we obtain: 


$$
\begin{aligned}
& \sum_{n t s=0}^{\infty}\left(R\left(n+t+s+\frac{3}{2}\right)-E\right) b_{n t s} H_{n}(x) H_{t}(y) H_{s}(z) \\
& -\sum_{n t s=0}^{\infty} R n(n-1) b_{n t s} H_{n-2}(x) H_{t}(y) H_{s}(z) \\
& -\sum_{n t s=0}^{\infty} R t(t-1) b_{n t s} H_{n}(x) H_{t-2}(y) H_{s}(z) \\
& -\sum_{n t s=0}^{\infty} R s(s-1) b_{n t s} H_{n}(x) H_{t}(y) H_{s-2}(z) \\
& -\sum_{n t s=0}^{\infty} \frac{R}{4} b_{n t s} H_{n+2}(x) H_{t}(y) H_{s}(z) \\
& -\sum_{n t s=0}^{\infty} \frac{R}{4} b_{n t s} H_{n}(x) H_{t+2}(y) H_{s}(z) \\
& -\sum_{n t s=0}^{\infty} \frac{R}{4} b_{n t s} H_{n}(x) H_{t}(y) H_{s+2}(z) \\
& +\sum_{n t s=0}^{\infty} \sum_{k=0}^{a b c} \sum_{f h r} c_{f n k} c_{h t l} c_{r s m} b_{n t s} u_{k l m} H_{f}(x) H_{h}(y) H_{r}(z)=0
\end{aligned}
$$

Suppose that we need to construct a matrix with the dimensions $(d+1)^{3} \times(d+1)^{3}$. We determine coefficients for the factor $H_{n^{\prime}}(x) H_{t^{\prime}}(y) H_{s^{\prime}}(z)$ :

$$
\begin{aligned}
& \left(R\left(n^{\prime}+t^{\prime}+s^{\prime}+\frac{3}{2}\right)-E\right) b_{n^{\prime} t^{\prime} s^{\prime}} H_{n^{\prime}}(x) H_{t^{\prime}}(y) H_{s^{\prime}}(z) \\
& -R\left(n^{\prime}+2\right)\left(n^{\prime}+1\right) b_{n^{\prime}+2, t^{\prime}, s^{\prime}} H_{n^{\prime}}(x) H_{t^{\prime}}(y) H_{s^{\prime}}(z) \\
& -R\left(t^{\prime}+2\right)\left(t^{\prime}+1\right) b_{n^{\prime}, t^{\prime}+2, s^{\prime}} H_{n^{\prime}}(x) H_{t^{\prime}}(y) H_{s^{\prime}}(z) \\
& \left.-R\left(s^{\prime}+2\right)\left(s^{\prime}+1\right)\right) b_{n^{\prime}, t^{\prime}, s^{\prime}+2} H_{n^{\prime}}(x) H_{t^{\prime}}(y) H_{s^{\prime}}(z) \\
& -\frac{R}{4} b_{n^{\prime}-2, t^{\prime}, s^{\prime}} H_{n^{\prime}}(x) H_{t^{\prime}}(y) H_{s^{\prime}}(z) \\
& -\frac{R}{4} b_{n^{\prime}, t^{\prime}-2, s^{\prime}} H_{n^{\prime}}(x) H_{t^{\prime}}(y) H_{s^{\prime}}(z) \\
& -\frac{R}{4} b_{n^{\prime}, t^{\prime}, s^{\prime}-2} H_{n^{\prime}}(x) H_{t^{\prime}}(y) H_{s^{\prime}}(z) \\
& +\sum_{n t s=0}^{d} \sum_{k l m} c_{n^{\prime} n k} c_{t^{\prime} t l} c_{s^{\prime} s m} b_{n t s} u_{k l m} H_{n^{\prime}}(x) H_{t^{\prime}}(y) H_{s^{\prime}}(z)=0
\end{aligned}
$$

This expression may be rewritten as follows:

$$
\begin{aligned}
& \left(R\left(n^{\prime}+t^{\prime}+s^{\prime}+\frac{3}{2}\right)-E\right) b_{n^{\prime} t^{\prime} s^{\prime}} H_{n^{\prime}}(x) \\
& -R\left(n^{\prime}+2\right)\left(n^{\prime}+1\right) b_{n^{\prime}+2, t^{\prime}, s^{\prime}}-R\left(t^{\prime}+2\right)\left(t^{\prime}+1\right) b_{n^{\prime}, t^{\prime}+2, s^{\prime}} \\
& -R\left(s^{\prime}+2\right)\left(s^{\prime}+1\right) b_{n^{\prime}, t^{\prime}, s^{\prime}+2}-\frac{R}{4} b_{n^{\prime}-2, t^{\prime}, s^{\prime}}-\frac{R}{4} b_{n^{\prime}, t^{\prime}-2, s^{\prime}} \\
& -\frac{R}{4} b_{n^{\prime}, t^{\prime}, s^{\prime}-2}+\sum_{n t s=0}^{d} \sum_{k l m} c_{n^{\prime} n k} c_{t^{\prime} t l} c_{s^{\prime} s m} b_{n t s} u_{k l m}=0
\end{aligned}
$$

We fix an order of the coefficients $b_{\text {nts }}$ in the column vector of the wave function defined by Equation (48). For example, if $d=2$, the transposed column vector may be of the form:

$$
\begin{aligned}
& \|\tilde{b}\|=\left\langle b_{0,0,0} ; b_{0,0,1} ; b_{0,0,2} ; b_{0,1,0} ; b_{0,1,1} ; b_{0,1,2} ; b_{0,2,0} ; b_{0,2,1} ;\right. \\
& b_{0,2,2} ; b_{1,0,0} ; b_{1,0,1} ; b_{1,0,2} ; b_{1,1,0} ; b_{1,1,1} ; b_{1,1,2} ; b_{1,2,0} ; b_{1,2,1} ; b_{1,2,2} ;(52) \\
& \left.b_{2,0,0} ; b_{2,0,1} ; b_{2,0,2} ; b_{2,1,0} ; b_{2,1,1} ; b_{2,1,2} ; b_{2,2,0} ; b_{2,2,1} ; b_{2,2,2}\right\rangle
\end{aligned}
$$

Let us assume that a change in three indices $\left(n^{\prime}, t^{\prime}, s^{\prime}\right)$ numbering rows of the Hamiltonian matrix is in the same order from top to bottom. The matrix element $H$ is numbered by a pair of three indices $H_{\left(n^{\prime}, t^{\prime}, s^{\prime}\right),(n, t, s)}$. As earlier, first we can fill the Hamiltonian matrix with the existing elements representing the potential energy of the form $c_{n^{\prime} n k} c_{t^{\prime} t l} c_{s^{\prime} s m} u_{k l m}$ by the following principle:

$$
H_{\left(n^{\prime}, t^{\prime}, s^{\prime}\right),(n, t, s)}=\sum_{k l m} c_{n^{\prime} n k} c_{t^{\prime} t l} C_{s^{\prime} s m} u_{k l m}
$$

Summation in Equation (53) is performed over all the existing triples $(k, l, m)$ for the pair of the specified triples $\left(n^{\prime}, t^{\prime}, s^{\prime}\right)$ and $(n, t, s)$. For the main diagonal $H_{\left(n^{\prime}, t^{\prime}, s^{\prime}\right),\left(n^{\prime}, t^{\prime}, s^{\prime}\right)}$ we must add $\left(n^{\prime}+t^{\prime}+s^{\prime}+\frac{3}{2}\right) R$. To the nondiagonal elements of the form $H_{\left(n^{\prime}, t^{\prime}, s^{\prime}\right),\left(n^{\prime}+2, t^{\prime}, s^{\prime}\right)}$; $H_{\left(n^{\prime}, t^{\prime}, s^{\prime}\right),\left(n^{\prime}+2, t^{\prime}, s^{\prime}\right)}$, and $H_{\left(n^{\prime}, t^{\prime}, s^{\prime}\right),\left(n^{\prime}, t^{\prime}, s^{\prime}+2\right)}$ we must add $-R\left(n^{\prime}+2\right)\left(n^{\prime}+1\right) ; \quad-R\left(t^{\prime}+2\right)\left(t^{\prime}+1\right)$, and $-R\left(s^{\prime}+2\right)\left(s^{\prime}+1\right)$. Finally, to the diagonal elements of the form $H_{\left(n^{\prime}, t^{\prime}, s^{\prime}\right),\left(n^{\prime}-2, t^{\prime}, s^{\prime}\right)} ; H_{\left(n^{\prime}, t^{\prime}, s^{\prime}\right),\left(n^{\prime}, t^{\prime}-2, s^{\prime}\right)}$, and $H_{\left(n^{\prime}, t^{\prime}, s^{\prime}\right),\left(n^{\prime}, t^{\prime}, s^{\prime}-2\right)}$ we must add $-\frac{1}{4} R$. Thus, we have:

$$
\begin{aligned}
H_{\left(n^{\prime}, t^{\prime}, s^{\prime}\right),\left(n^{\prime}, t^{\prime}, s^{\prime}\right)}= & \left(n^{\prime}+t^{\prime}+s^{\prime}+\frac{3}{2}\right) R \\
& +\sum_{k l m} c_{n^{\prime} n^{\prime} k} C_{t^{\prime} t^{\prime} l} C_{s^{\prime} s^{\prime} m} u_{k l m} \\
H_{\left(n^{\prime}, t^{\prime}, s^{\prime}\right),\left(n^{\prime}+2, t^{\prime}, s^{\prime}\right)} & =\sum_{k l m} c_{n^{\prime}, n^{\prime}+2, k} C_{t^{\prime} t^{\prime} l} C_{s^{\prime} s^{\prime} m} u_{k l m} \\
& -R\left(n^{\prime}+2\right)\left(n^{\prime}+1\right) \\
H_{\left(n^{\prime}, t^{\prime}, s^{\prime}\right),\left(n^{\prime}, t^{\prime}+2, s^{\prime}\right)} & =\sum_{k l m} c_{n^{\prime} n^{\prime} k} C_{t^{\prime}, t^{\prime}+2, l} C_{s^{\prime} s^{\prime} m} u_{k l m} \\
& -R\left(t^{\prime}+2\right)\left(t^{\prime}+1\right) \\
H_{\left(n^{\prime}, t^{\prime}, s^{\prime}\right),\left(n^{\prime}, t^{\prime}, s^{\prime}+2\right)}= & \sum_{k l m} c_{n^{\prime} n^{\prime} k} C_{t^{\prime} t^{\prime} l} c_{s^{\prime}, s^{\prime}+2, m} u_{k l m} \\
& -R\left(s^{\prime}+2\right)\left(s^{\prime}+1\right) \\
H_{\left(n^{\prime}, t^{\prime}, s^{\prime}\right),\left(n^{\prime}-2, t^{\prime}, s^{\prime}\right)} & =\sum_{k l m} c_{n^{\prime}-2, n^{\prime}, k} C_{t^{\prime} t^{\prime} l} C_{s^{\prime} s^{\prime} m} u_{k l m}-\frac{1}{4} R \\
H_{\left(n^{\prime}, t^{\prime}, s^{\prime}\right),\left(n^{\prime}, t^{\prime}-2, s^{\prime}\right)} & =\sum_{k l m} c_{n^{\prime} n^{\prime} k} C_{t^{\prime}, t^{\prime}-2, l} C_{s^{\prime} s^{\prime} m} u_{k l m}-\frac{1}{4} R \\
H_{\left(n^{\prime}, t^{\prime}, s^{\prime}\right),\left(n^{\prime}, t^{\prime}, s^{\prime}-2\right)} & =\sum_{k l m} c_{n^{\prime} n^{\prime} k} C_{t^{\prime} t^{\prime} l} C_{s^{\prime} s^{\prime} m} u_{k l m}-\frac{1}{4} R
\end{aligned}
$$

In other cases, we have (53). Now numbering the matrix elements of $H$ by the ordinary indices $(i, j)$ each of 
which is varying from 1 to $(2 d+1)^{3}$, we have to establish for them a one-to-one correspondence to three numbers according to the principle: $i \Leftrightarrow\left(n_{i}^{\prime}, t_{i}^{\prime}, s_{i}^{\prime}\right)$; $j \Leftrightarrow\left(n_{j}, t_{j}, s_{j}\right)$. Specifically, in the case given by (52) for $i=5$ we have $n_{5}^{\prime}=0 ; t_{5}^{\prime}=1 ; s_{5}^{\prime}=1$, and for $j=17$ we have $n_{17}=1 ; t_{17}=2 ; s_{17}=1$. Then an algorithm to construct the matrix $H_{i j}$ under condition of (52) takes the following form:

$$
\begin{aligned}
& H_{i i}=\left(n_{i}^{\prime}+t_{i}^{\prime}+s_{i}^{\prime}+\frac{3}{2}\right) R+\sum_{k l m} c_{n_{i}^{\prime} n_{i}^{\prime} k} c_{t_{i}^{\prime} t_{i}^{\prime} l_{i}} c_{s_{i}^{\prime} s_{i}^{\prime} m} u_{k l m} ; \\
& H_{i, i+18}=\sum_{k l m} c_{n_{i}^{\prime}, n_{i+18}^{\prime}, k} C_{t_{i}^{\prime} t_{i}^{\prime} l} c_{s_{i}^{\prime} s_{i}^{\prime} m} u_{k l m}-R\left(n_{i}^{\prime}+2\right)\left(n_{i}^{\prime}+1\right) ; \\
& i=1-9 \text {; } \\
& H_{i, i+6}=\sum_{k l m} c_{n_{i}^{\prime} n_{i}^{\prime} k} C_{t_{i}^{\prime}, t_{i+6}^{\prime}, l} c_{s^{\prime} s^{\prime} m} u_{k l m}-R\left(t^{\prime}+2\right)\left(t^{\prime}+1\right) ; \\
& i=1-3 ; 10-12 ; 19-21 \text {; } \\
& H_{i, i+2}=\sum_{k l m} c_{n_{i}^{\prime} n_{i}^{\prime} k} c_{t_{i}^{\prime} t_{i}^{\prime} l} c_{s_{i}^{\prime}, s_{i+2}^{\prime}, m} u_{k l m}-R\left(s^{\prime}+2\right)\left(s^{\prime}+1\right) ; \\
& i=1,4,7,10,13,16,19,22,25 \text {; } \\
& H_{i+18, i}=\sum_{k l m} c_{n_{i}^{\prime}, n_{i-18}^{\prime}, k} C_{t_{i}^{\prime} t_{i}^{\prime} l} C_{s_{i}^{\prime} s_{i}^{\prime} m} u_{k l m}-\frac{1}{4} R ; i=1-9 ; \\
& H_{i+6, i}=\sum_{k l m} c_{n_{i}^{\prime} n_{i}^{\prime} k} C_{t_{i}^{\prime}, t_{i-6}^{\prime}, l} c_{s_{i}^{\prime} s_{i}^{\prime} m} u_{k l m}-\frac{1}{4} R ; \\
& i=1-3 ; 10-12 ; 19-21 \text {; } \\
& H_{i+2, i}=\sum_{k l m} C_{n_{i}^{\prime} n_{i}^{\prime} k} C_{t_{i}^{\prime} t_{i}^{\prime} l} C_{s_{i}^{\prime} s_{i-2}^{\prime} m} u_{k l m}-\frac{1}{4} R \\
& i=1,4,7,10,13,16,19,22,25 \text {; } \\
& H_{i, j}=\sum_{k l m} C_{n_{i}^{\prime} n_{j} k} C_{t_{i}^{\prime} t_{j} l} C_{s_{i}^{\prime} s_{j} m} u_{k l m}
\end{aligned}
$$

\section{Conclusion}

In this way we have derived analytical expressions for elements of the Hamiltonian matrix describing the molecules characterized by motions with a large amplitude. The cases when the wave functions and potential energy are represented by Fourier series and orthogonal polynomials have been considered in detail taking Hermitian polynomials as an example. Some specific types of Schrödinger equations with a single variable or several variables have been treated.

\section{REFERENCES}

[1] J. T. Hougen, "A Group-Theoretical Treatment of Electronic, Vibrational, Torsional and Rotational Motions in the Dimethylacetylene Molecule," Canadian Journal of Physics, Vol. 42, No. 10, 1964, pp. 1920-1937. doi:10.1139/p64-182

[2] N. Moazzen-Ahmadi, H. P. Gush, M. Halpern, H. Jagannath, A. Leung and I. Ozier, "The Torsional Spectrum of $\mathrm{CH}_{3} \mathrm{CH}_{3}$," Journal of Chemical Physics, Vol. 88, No. 2, 1988, pp. 563-577. doi:10.1063/1.454183

[3] J. M. Fernandez-Sanchez, A. G. Valdenebro and S. Montero, "The Torsional Raman Spectra of $\mathrm{C}_{2} \mathrm{H}_{6}$ and $\mathrm{C}_{2} \mathrm{D}_{6}$," Journal of Chemical Physics, Vol. 91, No. 6, 1989, pp. 3327-3334. doi:10.1063/1.456908

[4] N. Moazzen-Ahmadi, A. R. W. McKellar, J. W. C. Johns and I. Ozier, "Intensity Analysis of the Torsional Spectrum of $\mathrm{CH}_{3} \mathrm{CH}_{3}$," Journal of Chemical Physics, Vol. 97, No. 6, 1992, pp. 3981-3988. doi:10.1063/1.462937

[5] K. S. Pitzer and W. D. Gwinn, "Energy Levels and Thermodynamic Functions for Molecules with Internal Rotation I. Rigid Frame with Attached Tops," Journal of Chemical Physics, Vol. 10, No. 7, 1942, pp. 428-440. doi:10.1063/1.1723744

[6] J. D. Swalen and D. R. Herschbach, "Internal Barrier of Propylene Oxide from the Microwave Spectrum," Journal of Chemical Physics, Vol. 27, No. 1, 1957, pp. 100108. doi:10.1063/1.1743645

[7] R. W. Kilb, C. C. Lin and E. B. Wilson, "Calculation of Energy Levels for Internal Torsion and Over-All Rotation. II. $\mathrm{CH}_{3} \mathrm{CHO}$ Type Molecules; Acetaldehyde Spectra," Journal of Chemical Physics, Vol. 26, No. 6, 1957, pp. 1695-1703. doi:10.1063/1.1743607

[8] R. Subramanian, S. E. Novick and R. K. Bohn, "Torsional Analysis of 2-Butynol," Journal of Molecular Spectroscopy, Vol. 222, No. 1, 2003, pp. 57-62. doi:10.1016/S0022-2852(03)00170-X

[9] D. A. Shea, L. Goodman and M. G. White, "Acetone $n$-Radical Cation Internal Rotation Spectrum: The Torsional Potential Surface," Journal of Chemical Physics, Vol. 112, No. 6, 2000, pp. 2762-2768. doi:10.1063/1.480850

[10] E. V. Ivash and D. M. Dennison, "The Methyl Alcohol Molecule and Its Microwave Spectrum," Journal of Chemical Physics, Vol. 21, No. 10, 1953, pp. 1804-1816. doi:10.1063/1.1698668

[11] K. T. Hecht and D. M. Dennison, "Hindered Rotation in Molecules with Relatively High Potential Barriers" Journal of Chemical Physics, Vol. 26, No. 1, 1957, pp. 31-47. doi:10.1063/1.1743262

[12] G. A. Pitsevich and M. Shundalau, "Computer Simulation of the Effect Exerted by Argon Matrix on the Internal Rotation Barriers and Torsional States of Methanol Molecule" Journal of Spectroscopy and Dynamics, Vol. 2, No. 3, 2012, p. 15 http://www.simplex-academic-publishers.com/jsd.aspx 